\title{
Langzeitauswertung: Chemotherapie überzeugt bei anaplastischem Gliom
}

\author{
In der NOA-04-Studie war beim anaplastischen Gliom eine Chemotherapie \\ nach der Operation der Radiatio ebenbürtig. Jetzt wurde geschaut, ob das \\ 10 Jahre nach dem Eingriff noch immer gilt.
}

n der 2009 veröffentlichten NOA04-Studie wurde bei 318 Patienten mit einem anaplastischen Gliom vom Grad III postoperativ eine Radiotherapie mit einer Chemotherapie verglichen. Letztere erfolgte entweder mit Temozolomid (TMZ) oder nach dem PCV(Procarbazin, CCNU, Vincristin)-Schema. Kam es zur Tumorprogression oder wurde die erste Therapiemodalität nicht ausreichend vertragen, wechselten die Ärzte auf die jeweils andere Modalität.

Nach maximal 4,5 Jahren war es weitgehend egal, ob die Ärzte mit Radiooder Chemotherapie begonnen hatten. Die Zeit bis zum Therapieversagen, das progressionsfreie Überleben (PFS) mit der ersten Therapie und das Gesamtüber- leben waren in allen Gruppen vergleichbar, auch gab es keine signifikanten Unterschiede zwischen einer Behandlung mit PCV oder dem besser verträglichen TMZ. Daher sprach viel dafür, nach der Operation mit TMZ zu beginnen.

Inzwischen liegt eine Auswertung nach median 9,5 Jahren vor, die im Wesentlichen die primäre Analyse bestätigte: $\mathrm{Zu}$ einer Progression kam es mit Radiatio als erster Option bei $78 \%$ der Patienten, mit der Chemotherapie bei $79 \%$. Ein Therapieversagen stellten die Ärzte bei 66 bzw. $67 \%$ der Patienten nach im Median 4,4 bzw. 4,6 Jahren fest. Auch beim Gesamtüberleben fanden sich keine statistisch signifikanten Unterschiede (median 8 vs. 6,5 Jahre; $\mathrm{p}=0,53$ ), wenn- gleich die Rate mit Radiatio als erster Therapiewahl nach der Operation relativ betrachtet um $11 \%$ höher lag.

In den Chemotherapiegruppen gab es insgesamt keine statistisch belastbaren Unterschiede beim PFS zwischen einer Behandlung mit PCV (2,5 Jahre) und TMZ (2,7 Jahre). Patienten mit GliomCpG-Inseln-Methylierungs-Phänotyp (G-CIMP) und 1p/19q-Kodeletion lebten jedoch mehr als doppelt so lange progressionsfrei, wenn sie PCV statt TMZ erhielten (9,4 vs. 4,5 Jahre). Auch die Radiatio war hier TMZ deutlich überlegen.

Fazit: Eine Chemotherapie wirkt bei Patienten mit anaplastischem Gliom nach der Operation zwar ähnlich gut wie die Bestrahlung, mit der Radiatio ist das Gesamtüberleben aber tendenziell besser. Auch kann TMZ nicht in allen prognostischen Subgruppen PCV ersetzen.

Thomas Müller

Wick W. et al. Long-term analysis of the NOA-04 randomized phase III trial of sequential radiochemotherapy of anaplastic glioma with PCV or temozolomide. Neuro Oncol. 2016;18(11):1529-37.

\section{Glioblastom: Ein Drittel mehr Lebenszeit}

\section{Eine Strahlentherapie in Kombination mit Temozolomid wurde bei älteren Patienten mit Glioblastom bislang nicht explizit geprüft. In einer aktuellen Studie wurde dies nachgeholt.}

Fis ür die Studie wurden 562 Patienten mit Glioblastom (mindestens 65 Jahre) entweder mit einer hypofraktionierten Radiatio alleine (erweiterte Tumorregion, $40 \mathrm{~Gy}$ in 15 Fraktionen über 2 Wochen) oder zusätzlich mit Temozolomid (TMZ) behandelt. TMZ (75 mg/m²) wurde konsekutiv für 21 Tage zeitgleich zur Radiotherapie sowie adjuvant $\left(150-200 \mathrm{mg} / \mathrm{m}^{2}\right.$; konsektuiv an 5 von 28 Tagen) für bis zu 12 Zyklen bzw. bis zur Tumorprogression verabreicht. $47 \%$ der Patienten hatten einen methylierten MGMT-Promotor.

Es ergaben sich deutliche Vorteile zugunsten der Kombinationstherapie: So lag das mediane Gesamtüberleben (OS) bei 9,3 Monaten mit Radiatio plus TMZ, mit alleiniger Bestrahlung nur bei 7,6 Monaten (Hazard Ratio [HR] für Tod 0,67; $\mathrm{p}<0,001)$. Beim progressionsfreien
Überleben waren es 5,3 versus 3,9 Monate. Interessanterweise profitierten über 75-Jährige hinsichtlich des OS am stärksten von der Kombination (10,0 vs. 7,1 Monate), die 65- bis 70-Jährigen differierten hingegen nicht einmal um einen halben Monat (8,7 vs. 8,3 Monate). Möglicherweise hätten diese Patienten doch eher von der bei jüngeren Patienten üblichen Strahlendosis (60 Gy in 30 Fraktionen) profitiert, spekulieren die Forscher. Vielleicht handelte es sich hier aber auch um eine Auswahl von Patienten mit besonders schlechtem Gesundheitszustand und damit sehr ungünstiger Prognose.

Wie erwartet überlebten Patienten mit methyliertem MGMT-Promotor mit 13,5 versus 7,7 Monaten unter der Kombinationstherapie etwa doppelt so lange. Doch auch die übrigen Patienten schie- nen tendenziell von der Kombination zu profitieren, wenngleich mit 10,0 versus 7,9 Monaten deutlich weniger.

Bei der Lebensqualität unterschieden sich die beiden Gruppen kaum, allerdings klagten die Patienten unter der Kombination etwas häufiger über Übelkeit und Verstopfung, auch traten Nebenwirkungen vom Grad 3/4 deutlich häufiger auf. So wurde eine Lymphopenie vom Grad $3 / 4$ bei $27 \%$ mit Kombi- und $10 \%$ mit Strahlentherapie beobachtet; Thrombozytopenien (11,1 vs. $0,4 \%$ ) und Neutropenien $(8,3$ vs. $0,8 \%)$ von Grad 3/4 waren unter der Kombination deutlich häufiger.

Fazit: Insgesamt scheint die Radiatio in Kombination mit TMZ auch bei älteren Patienten von Vorteil zu sein. Vor allem über 70-Jährige und Betroffene mit methyliertem MGMT-Promotor profitieren von der verkürzten Radiatio plus Chemotherapie. Thomas Müller

Perry JR et al. Short-Course Radiation plus Temozolomide in Elderly Patients with Glioblastoma. N Engl J Med. 2017;376(11):1027-37. 